\title{
An Automated Tabletop Continuous Culturing system with Multicolor Fluorescence Monitoring for Microbial Gene Expression and Long-Term Population Dynamics
}

Ue-Yu Pen ${ }^{1}$, Christopher J. Nunn ${ }^{1}$, and Sidhartha Goyal ${ }^{1,2}$

${ }^{1}$ Department of Physics and ${ }^{2}$ IBBME, University of Toronto, Ontario, Canada, M5S 1A7

\section{S-I. Additional figures}

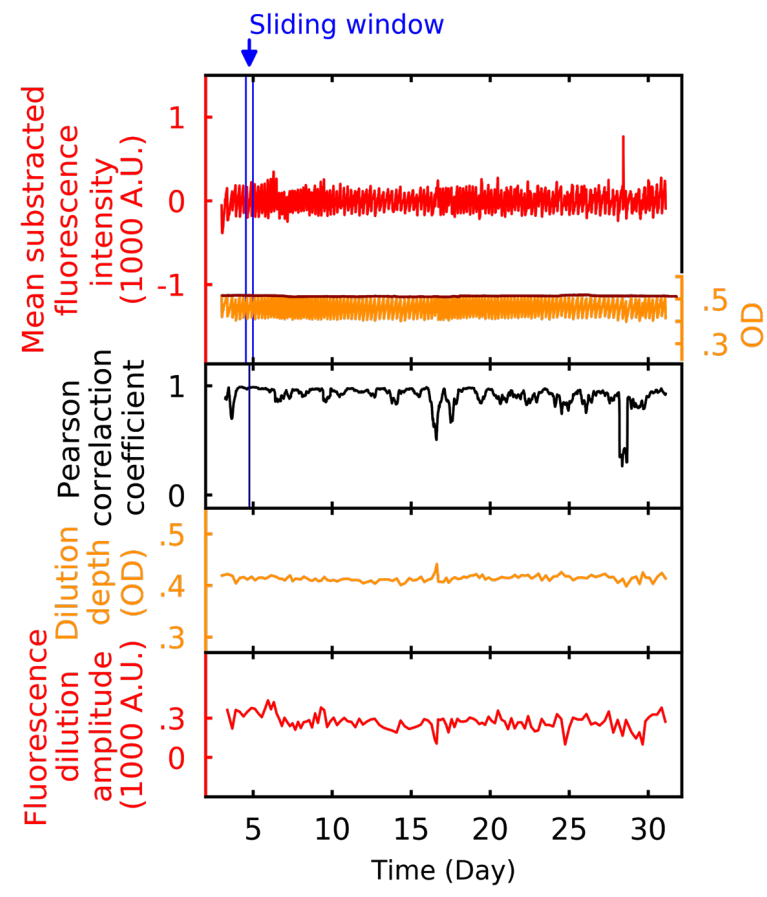

\section{Figure S1. Investigating biofilm formation.}

Previous studies have thoroughly investigated the ability of similar continuing device designs to maintain stable environmental conditions. Besides hardware/software interruptions, biofilm formation remains a concern for continuous culturing devices. To investigate the biofilm formation in our platform, figure S1 is plotted. First panel: A 36 day experiment where a bacterial population is maintained at a constant turbidity (OD in orange) with fluorescence measurements (red) taken in parallel. The fluorescence signal is meansubtracted and treated with a high pass filter to focus on the potential issue of biofilm formation in our fluorescence measurement probe. This processing eliminates any drift due to changes in fluorophore expression, emphasizing the effect of turbidity maintaining operation. Second panel: Correlation between the fluorescence signal and OD throughout the experiment which are highly correlated. Third panel: The minimum OD observed during each dilution cycle. Fourth panel: Amplitude of the fluorescence signal across dilution cycles. If biofilm was accumulating near the observation window of the probe, fluorescence measurements taken via the probe could end up decoupling from the cycling OD due to occlusion of the observation window. This occlusion may also cause the amplitude of these cycles to decrease, as biofilm itself may fluoresce in addition to blocking fluorescence of the culture behind it. We do not see any indication of probe occlusion in these plots. The lower limit of OD in these dilution cycles shown in the third panel one would expect to drift if biofilm was accumulating on the walls of the vial. Again, we see no indication of this, which highlights the ability of our device to culture over long periods with appropriate selection of strains and target optical densities. 

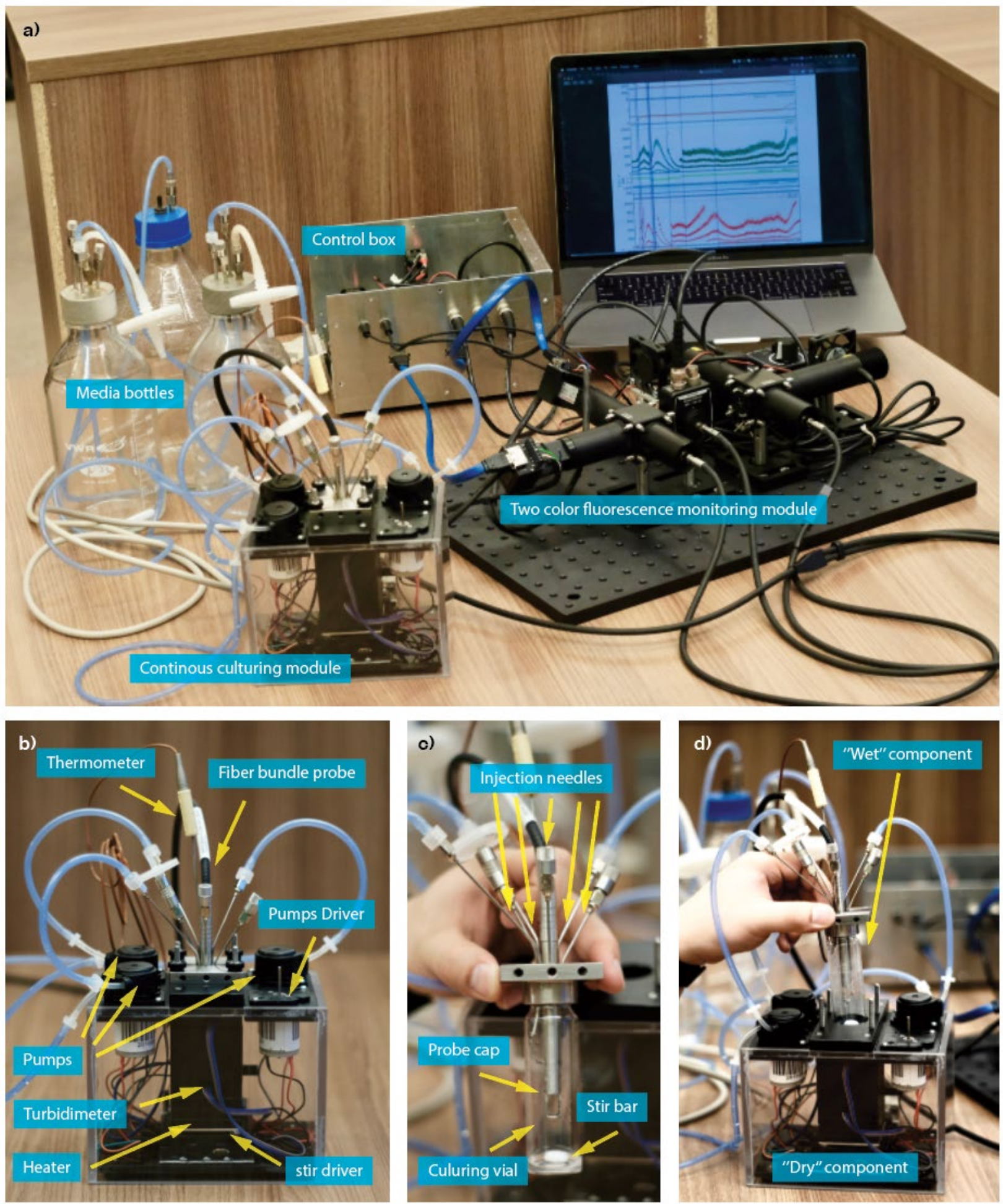

\section{Figure S2. Details of the experimental setup.}

a) Overview of the system showing the culturing module fed by media bottles and the control box which controls the two color fluorescence monitoring module. b) The culturing module and its main components. c) A view of the culturing vial with stir bar and various liquid ports integrated in the custom designed cap. d) A custom made control box driven by an Arduino microcontroller. 


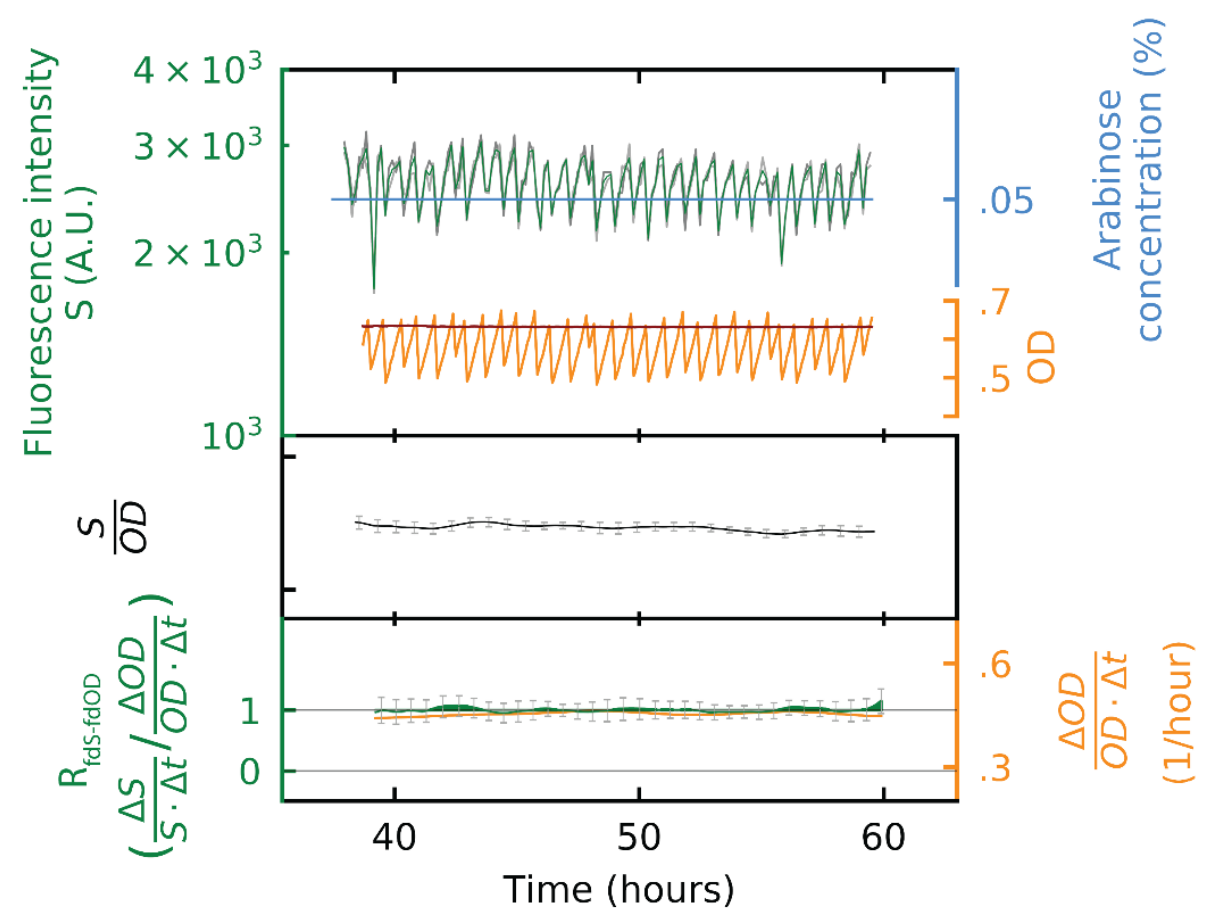

Figure S3. OD and fluorescence measurements of culture under steady state conditions.

Top panel: The orange curve shows the OD as measured by the turbidimeter on our continuous culturing device and the turbidity target $(\mathrm{OD}=0.6)$ is marked as the dark red line. Green (and technical repeats in grey) curve represent the fluorescence signal obtained from the green channel of the fluorescence monitoring module. Blue curve shows the arabinose concentration in the media. Middle panel: the fluorescence intensity (S) divided by turbidity (OD), indicates the averaged protein expression level per cell, and error bars represent the standard deviation across technical replicates. Bottom panel: the green curve shows the ratio of fold change of fluorescence intensity to the fold change of OD $\mathrm{R}_{\mathrm{fds}-\mathrm{fdOD}}$, with error bars across technical repeats. As the arabinose concentration is fixed during the experiment, the S/OD plot does not demonstrate the saw-tooth pattern seen in both the OD and fluorescence measurements. This indicates the average fluorescence intensity per cell $\mathrm{S} / \mathrm{OD}$ has reached a steady state with $\mathrm{R}_{\mathrm{fds}-\mathrm{fdOD}}=1$. The bacterial population growth rate (orange curve) is also stable. 

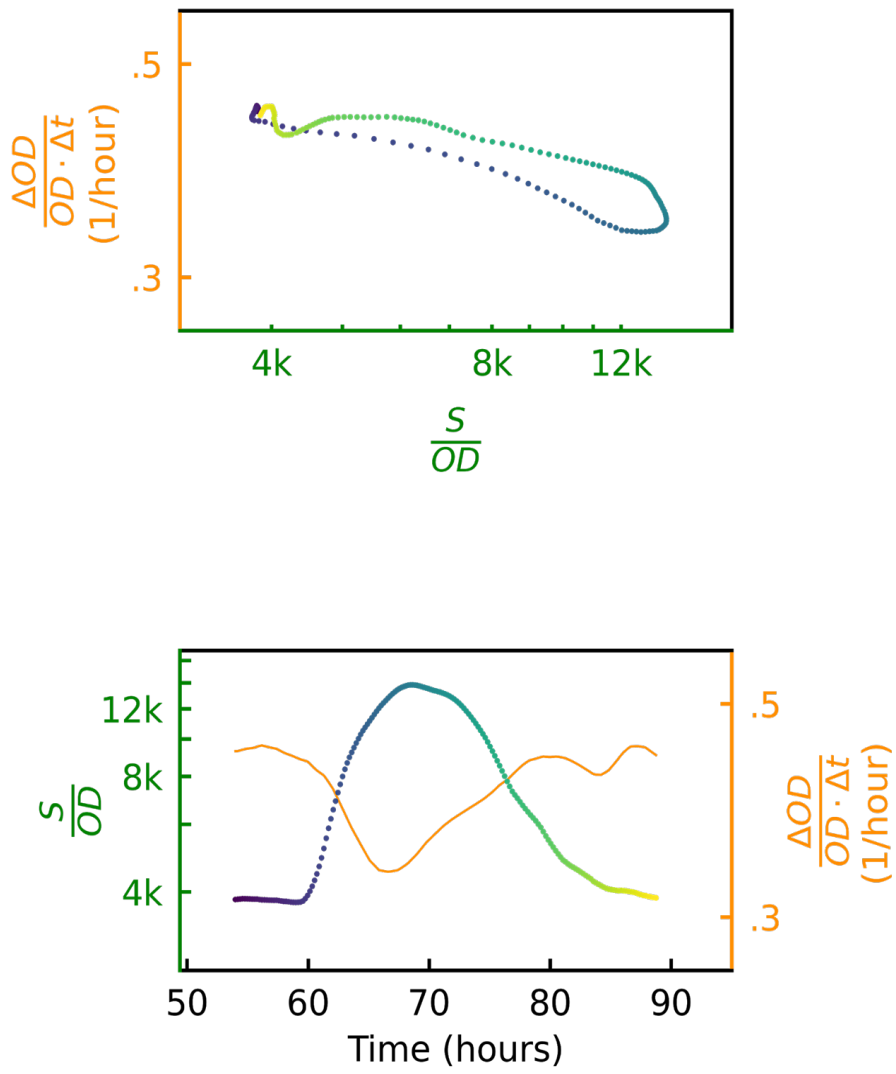

Figure S4. The response of growth rate to the change of fluorescence expression level.

The upper panel shows how the growth rate changes in response to the change of fluorescent protein expression of the sample, replotted from the data in the bottom panel. The colormap shows the data at the corresponding time. The figure shows that the growth rate decreases when the sample is expressing more fluorescent protein, indicating that this fluorescent protein is metabolically costly. The looped shape of this plot is due to the asymmetric response of the changes in growth rate to the changes in expression level shown in the bottom panel. On average, the culture growth rate is lower at early times, and higher at later times when the culture is exhibiting similar per cell fluorescence. This indicates that the same level of fluorescence is less costly at later times. 


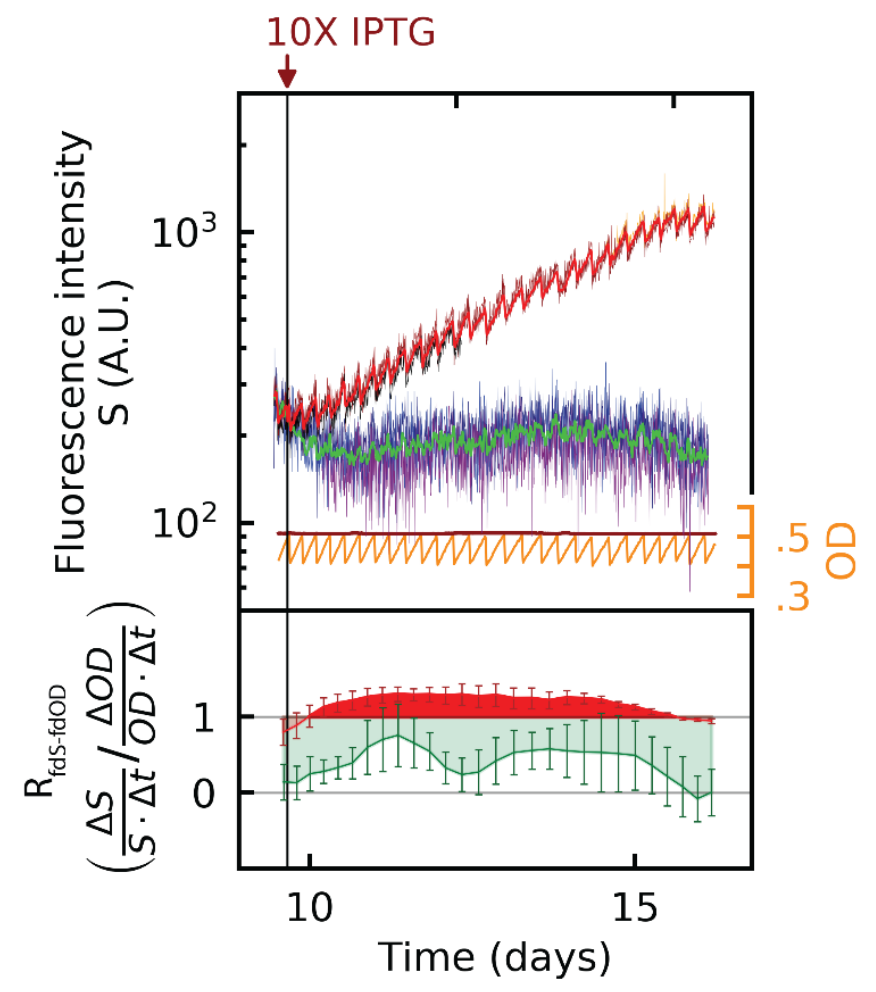

Figure S5. Results of 10X IPTG induction of pkDL071 toggle switch.

Top panel: Green (and technical repeats) and red (and technical repeats) curve show the fluorescence intensity measured by the green and the red channel of the two-fluorophore monitoring module for GFP and mCherry signals, respectively. The orange curve is the OD reading, and the turbidity target (OD $=0.5)$ is marked as the dark red line. Bottom panel: Green and red curves represent the ratios of fold change of the fluorescence intensity to the fold change of OD, $\mathrm{R}_{\mathrm{fds}-\mathrm{fdOD}}-\mathrm{GFP}$ and $\mathrm{R}_{\mathrm{fds}-\mathrm{fdOD}}-\mathrm{mCherry}$, with error bars representing the standard deviation across technical repeats. The dark red arrow shows the time of addition

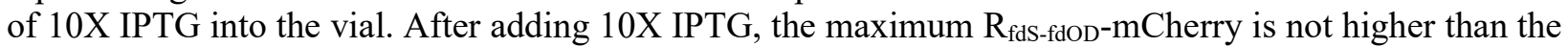
1X IPTG case shown in the Figure $3 \mathrm{c}$ in the main text. The effect of adding more IPTG only prolonged this elevated $\mathrm{R}_{\mathrm{fdS}-\mathrm{fdOD}}-\mathrm{mCherry}$, as more dilutions were required to dilute IPTG to a level that resulted in the

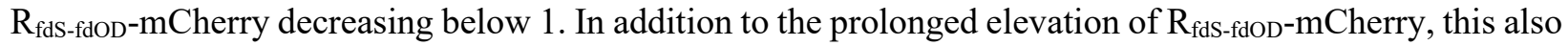
results in higher per cell fluorescence due to the continual accumulation of mCherry during this period. 


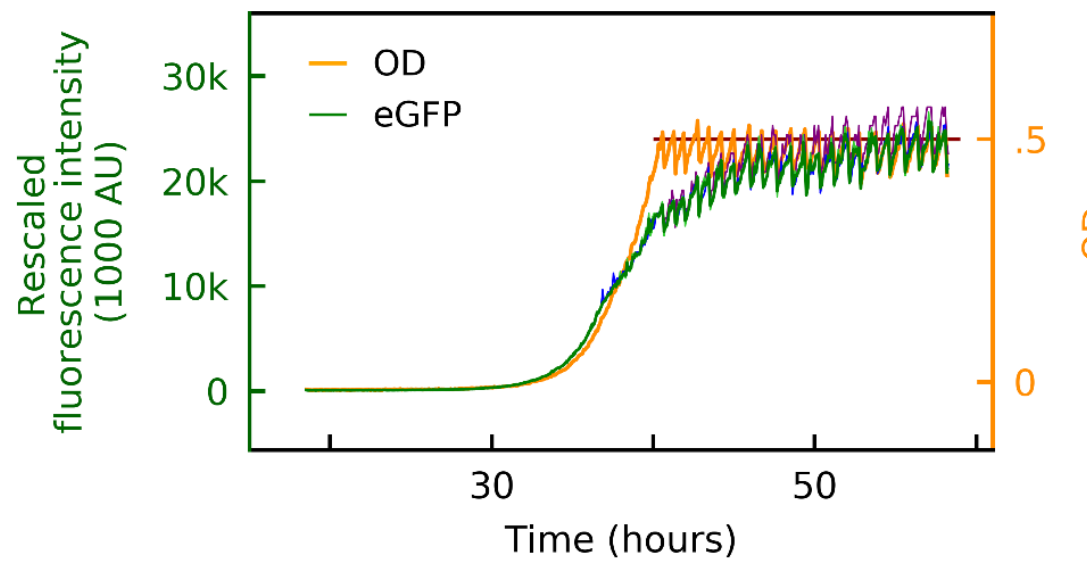

Figure S6. Lag of fluorescence expression level compared to the turbidity level during exponential growth and high density.

The green (and technical repeats) curve represents the eGFP fluorescence signal measured by our fluorescence monitoring module. The orange curve shows the turbidity of the sample and the dark red line indicates the turbidity target $(\mathrm{OD}=0.5)$. We used the BW25113 strain containing pBAD/eGFP for this experiment. A $0.5 \%$ working concentration of arabinose is added in both the culture and the turbidity maintaining dilution media. This figure shows a lag of fluorescence signal to the OD measurement after OD crosses around the value of 0.3 (corresponding to around $2.5^{*} 10^{8}$ cell $/ \mathrm{ml}$ ). However, after new media is injected in the culturing device to maintain OD the fluorescence level again aligns with the OD measurement. Taken together, this suggests either depletions of certain essential nutrient sources or/and the accumulation of unfavorable metabolic byproducts as cell density increases, and injection of fresh media that removes these unfavorable conditions for fluorescent protein expression. 


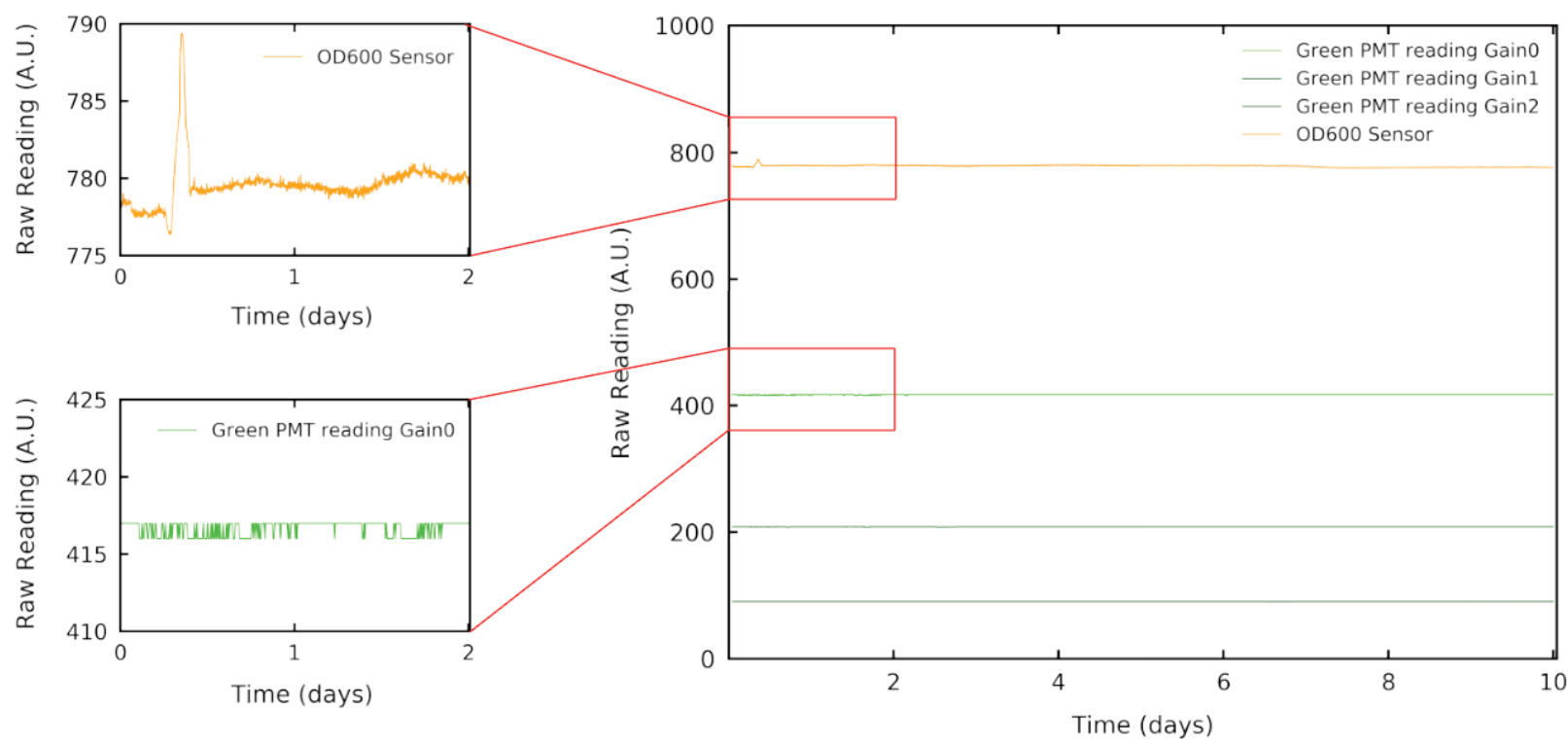

Figure S7. Drift of OD and fluorescence measurements within an experiment.

This figure shows a 10 day long experiment conducted with only M9 media in the vial. With this experiment, any instrumental drift can be seen while the sample is unchanging. As we can see in the figure, the raw OD reading and PMT readings under different gains are shown to be stable and no significant drift is observed. This experiment demonstrates that there is no significant drift in signal due to hardware instability. 


\section{S-II. Design of the adaptive amplification system for the fluorescence monitoring module}

In this work we utilize a micro photomultiplier tube (microPMT) package that converts light signal to the electrical signal extracted in fluorescence measurements. The converted electrical signal is sent to an analog to digital converter (AD converter) on the Arduino Mega 2560 controller for further processing.

Designed to detect low intensity light signals, microPMTs amplify the light by $10^{4}$ to $10^{7}$ fold during its conversion to electrical signals. This level of amplification is called PMT gain and depends directly on the voltage applied to the photomultiplier tube. The intensity of the excitation LED (regulated by LED gain) also affects the signal intensity received by the PMT of the sample. Given that the fluorescence signal changes in an entire population dynamics or/and gene expression experiment easily cross 6 orders of magnitudes or even more, proper selection of the gain levels for the PMT and LED based on the current output signal level is necessary to provide optimal performance of the fluorescence monitoring module. For example, when the applied voltage of the PMT (and thus the PMT gain) is properly selected, it keeps the change in output electrical signal in a range that the Arduino and the processing circuit can interpret, and allows the Arduino's analog to digital converter (AD converter) to resolve the signal dynamics. In contrast, without properly selecting the applied voltage (and thus again the PMT gain) of the PMT, when the fluorescence signal is weak, the electrical signal outputted by the PMT may become too weak to travel through the processing circuit, to be resolved by the $\mathrm{AD}$ converter, or to be resolved above the electrical noise of the circuit. On the other hand, when the fluorescence intensity is high, the output of the electrical signal may saturate the PMT output.

To automatically select the proper gain levels for the PMT and LED based on the current output signal level, we built an adaptive amplification system which contains a feedback control algorithm to actively regulate the PMT gain and the LED gain based on the intensity of the current electrical signal.

When first receiving the fluorescence signal, our system amplifies the incoming signal with a range of PMT gains. Different sets of amplified electrical signals generated by different PMT gains are then sent to the AD converter for analog to digital conversion. Our algorithm examines these AD converted digital data to decide which PMT gains are more suitable for this fluorescence signal level, and uses these set of PMT gains for further recordings. This algorithm actively monitors the incoming digital data for the entire experiment. Once the algorithm decides that the ongoing set of PMT gains no longer fit the current fluorescence signal level, it increases or decreases the PMT gains accordingly. When recording the converted digital data, every value is assigned with a prefix which indicates the PMT gain used while taking that measurement. After the experiment, the time series of the intensity of the fluorescence signal is reconstructed by using previously calibrated multipliers that allows direct comparison of the signal values acquired by different PMT gains (see table S1). We typically use a set of three PMT gains for the measurements, which allows at least one gain overlap between the adjacent PMT gain sets to cross check and improve signal reconstruction. See Figure S8 for a processing example.

Another tunable amplification parameter of the adaptive amplification system is the LED gain of the excitation LED driver. This parameter changes the intensity of the excitation light for the fluorescent protein. With a fixed number of fluorescent proteins, higher excitation intensity yields higher total fluorescence intensity. However, different levels of the excitation light may have different effects on the sample. For example, too strong excitation light damages the fluorescent proteins and causes photobleaching, resulting in the decay of fluorescence signals over time. When fluorescence signals serve as a proxy for the abundance of a protein of interest this could result in a significant discrepancy. In addition, different excitation intensities result in different rates of photobleaching. To avoid these uncertainties, LED gains used in all the experiments shown in this paper are fixed. However, when working in a proper LED intensity range and/or with a proper calibration, the ability to tune the LED gain in addition to the PMT gain could provide even wider dynamic range. In addition, in our system changing the LED gain results in a linear change in the amplification of the signal while changing the PMT gain results in an exponential amplification. Therefore altering the LED gain could provide more precise control for experiments 
requiring fine tuning of amplification levels.

To enable the use of different sets of LED gain and PMT gain interchangeably when measuring the same system, a 2-dimensional multiplications relationship map between different combinations of LED and PMT gains are needed. Figure S9 shows a map of raw $\mathrm{AD}$ converted digital readings when scanning a sample with a fixed amount of fluorescent proteins using different combinations of PMT and LED gains. This map gives us an idea of how these combinations relate to each other. One can see "contours" on this map, indicating that some combinations share similar amplifications, which implies under certain conditions the PMT gains and LED gains are interchangeable. To test if these multiplication relationships are universal, we constructed maps by using samples with different amounts of fluorescent proteins, and comparing them by normalizing the map with the reading generated by a certain gain combination. For example, maps in Figure S9 are generated when measuring the samples with different population sizes. By dividing the whole map by the reading generated by the combination LED gain $=140$, PMT gain $=43$ (green box in Figure S9 and the orange boxes in Figure S10) in the corresponding map, two maps become comparable and show a similar value in the map. We tested around 20 different samples from pure M9 to a population size $\sim 10^{8.5}$ and the normalized maps showed similar multiplication relationships between trials. This result indicates that the multiplication relationship between different PMT and LED gains combinations are robust when measuring different levels of fluorescence signal.

Besides, when we integrated the reads gathered from different samples into a map of log raw readings vs log populations size, we found that the readings are linearly dependent on sample population size, and hence the fluorescence intensity (shown in Figure S11). This makes it easy to find the multiplication relations between two PMT and LED gain sets by simply fitting two lines to each other. 


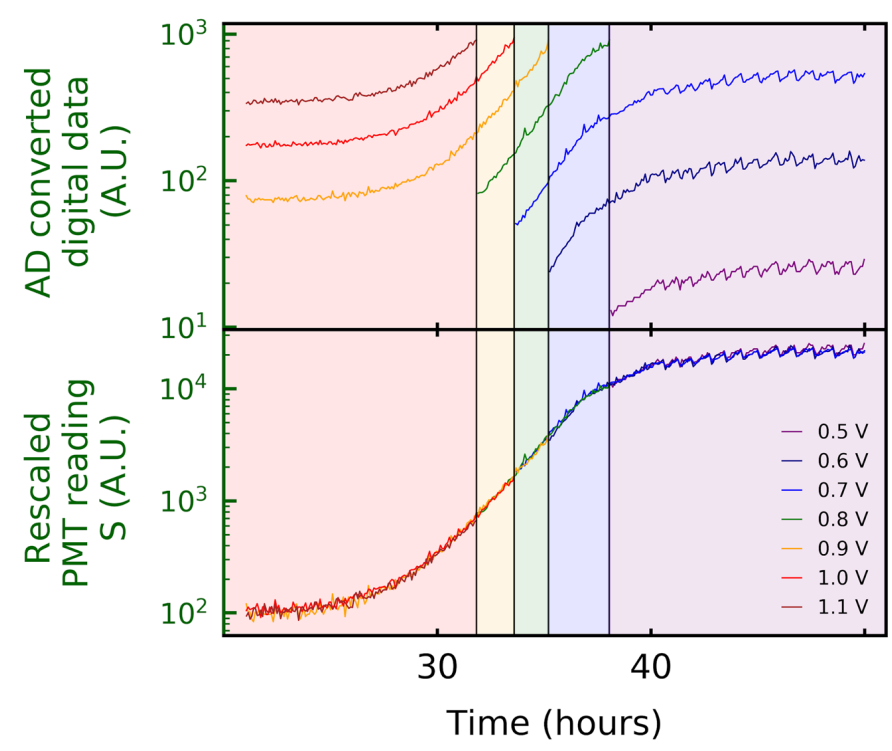

Figure S8. An example of the adaptive amplification system operation.

Colored curves show the data gathered under different PMT gains, with the control voltages setting labeled in the legend. Different colored areas indicate the time periods that common PMT gain sets were used to scan the sample. The top panel shows the raw readings right after the conversion of the AD converter while the bottom panel shows the reconstructed fluorescent signal by using previously calibrated multiplication relations between different PMT gains. 


\begin{tabular}{|l|c|c|c|c|c|c|c|}
\hline Control Voltage & $1.1 \mathrm{~V}$ & $1.0 \mathrm{~V}$ & $0.9 \mathrm{~V}$ & $0.8 \mathrm{~V}$ & $0.7 \mathrm{~V}$ & $0.6 \mathrm{~V}$ & $0.5 \mathrm{~V}$ \\
\hline Green channel & 1 & 2 & 4.6 & 12.5 & 41 & 178 & 1100 \\
\hline Red channel & 1 & 2 & 4.6 & 12.2 & 37 & 138 & 690 \\
\hline
\end{tabular}

Table S1. Multiplication relations by fold of different PMT gains to the maximum PMT gain (control voltage $=1.1)$ 


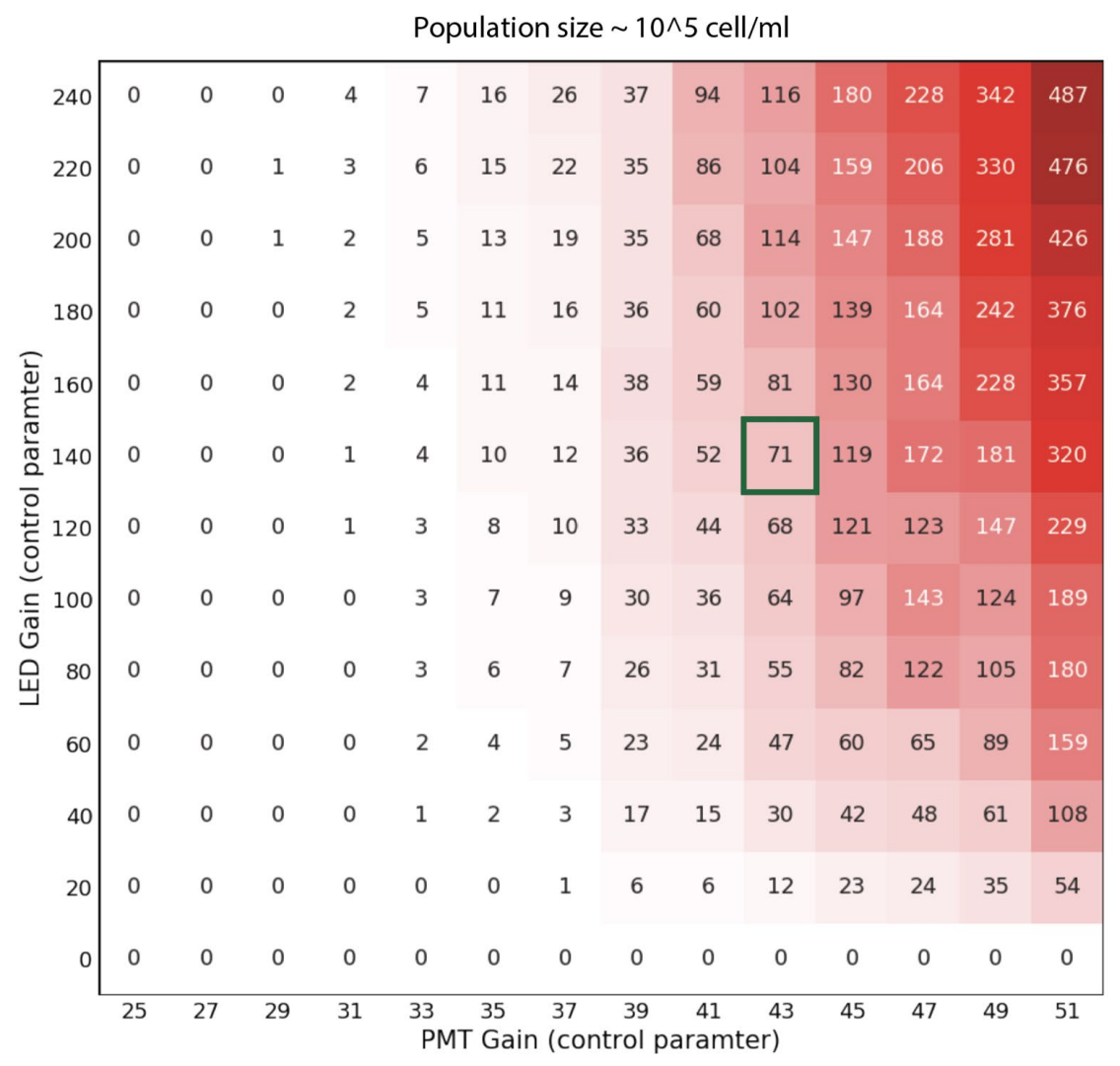

Figure S9. Map of the AD converted digital data readings generated by using different combinations of PMT and LED gains to measure a fluorescent sample (population size $\sim 10^{5}$ cells $/ \mathbf{m l})$. 
Population size $\sim 10 \wedge 5 \mathrm{cell} / \mathrm{ml}$

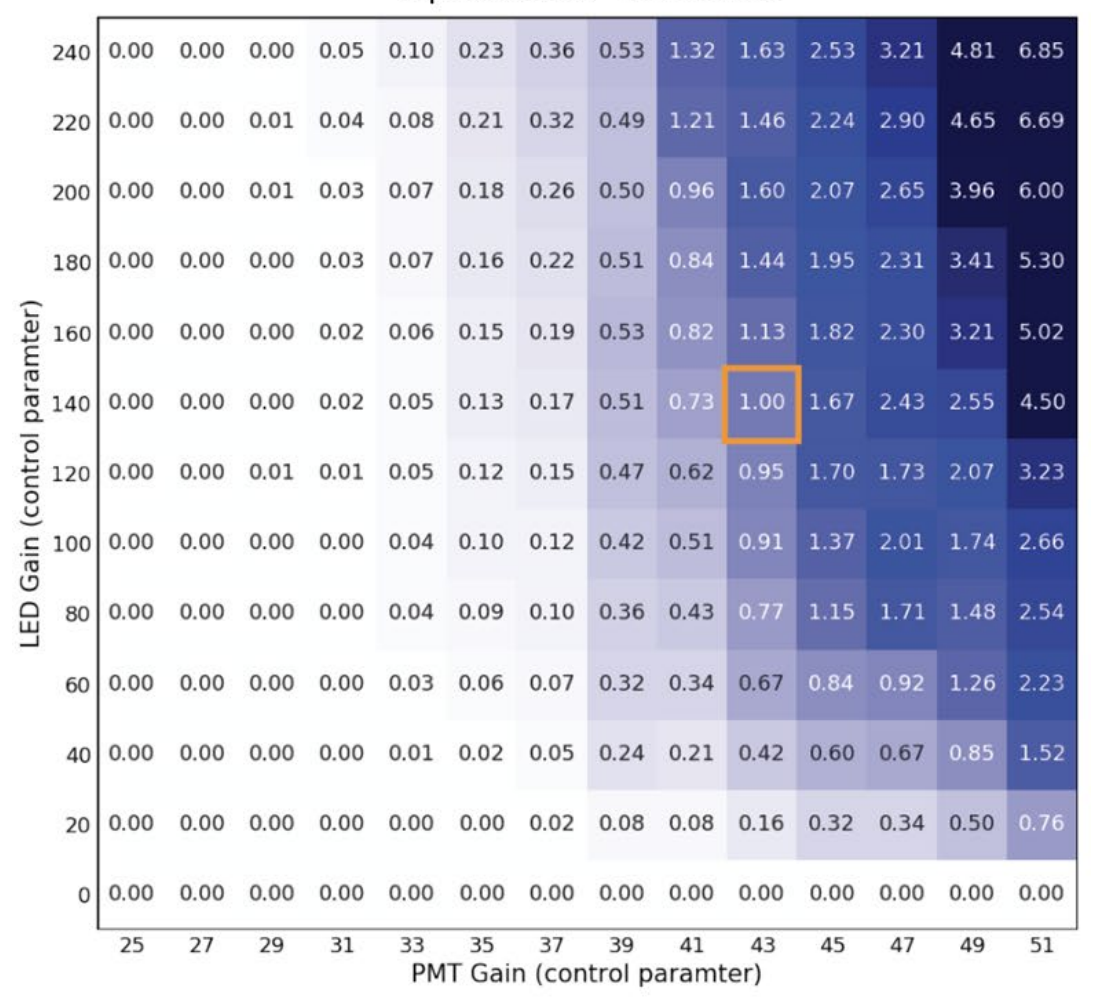

Population size $\sim 10 \wedge 4.5 \mathrm{cell} / \mathrm{ml}$

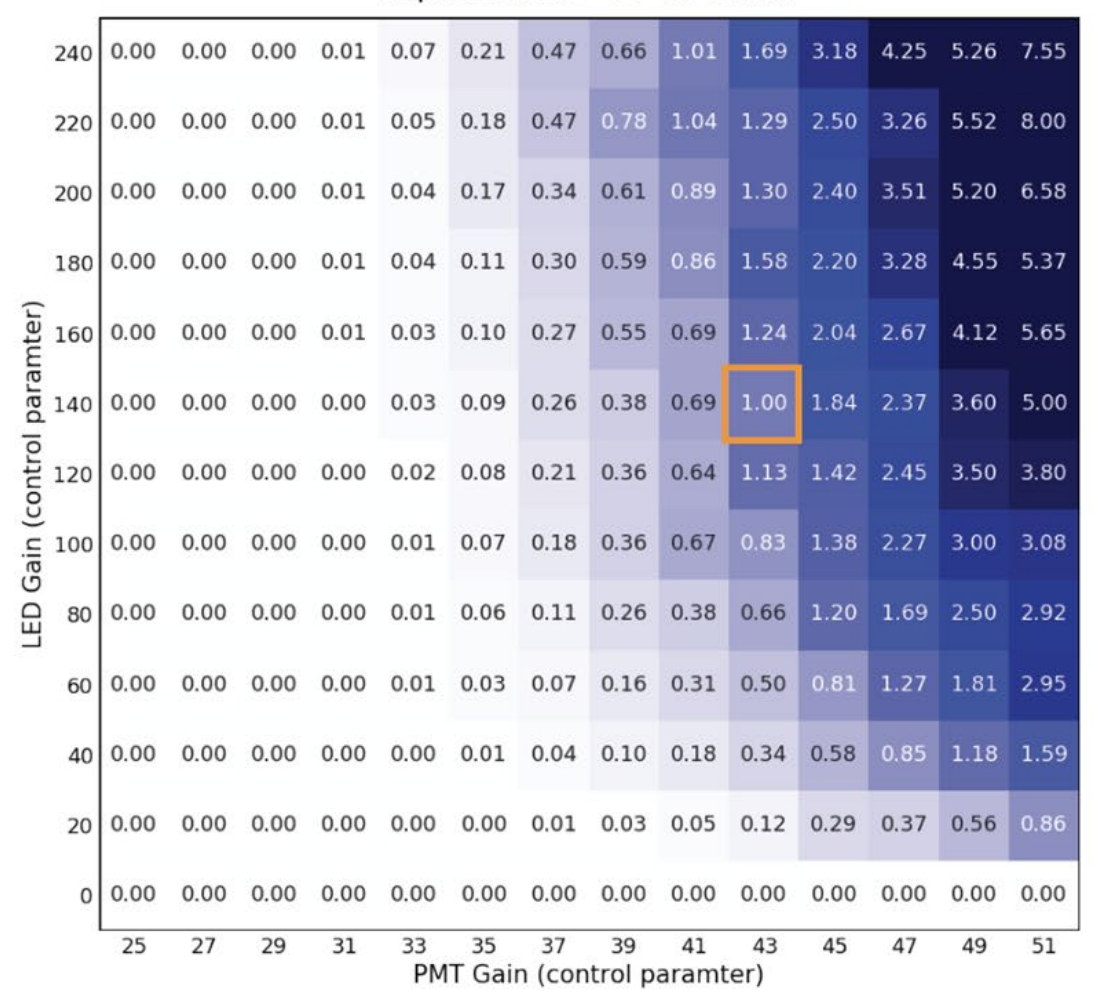


Figure S10. Normalized maps of signals measured by using different combinations of PMT and LED gains to measure different fluorescent samples. Upper panel: population size $\sim 10^{5}$ cells $/ \mathrm{ml}$. Lower panel: population size $\sim 10^{4.5}$ cells $/ \mathrm{ml}$. Values in the maps are normalized by the reading measured by the combination LED gain $=140$, PMT gain $=43$, as shown in the orange boxes in this figure and the green box in Figure S9. 


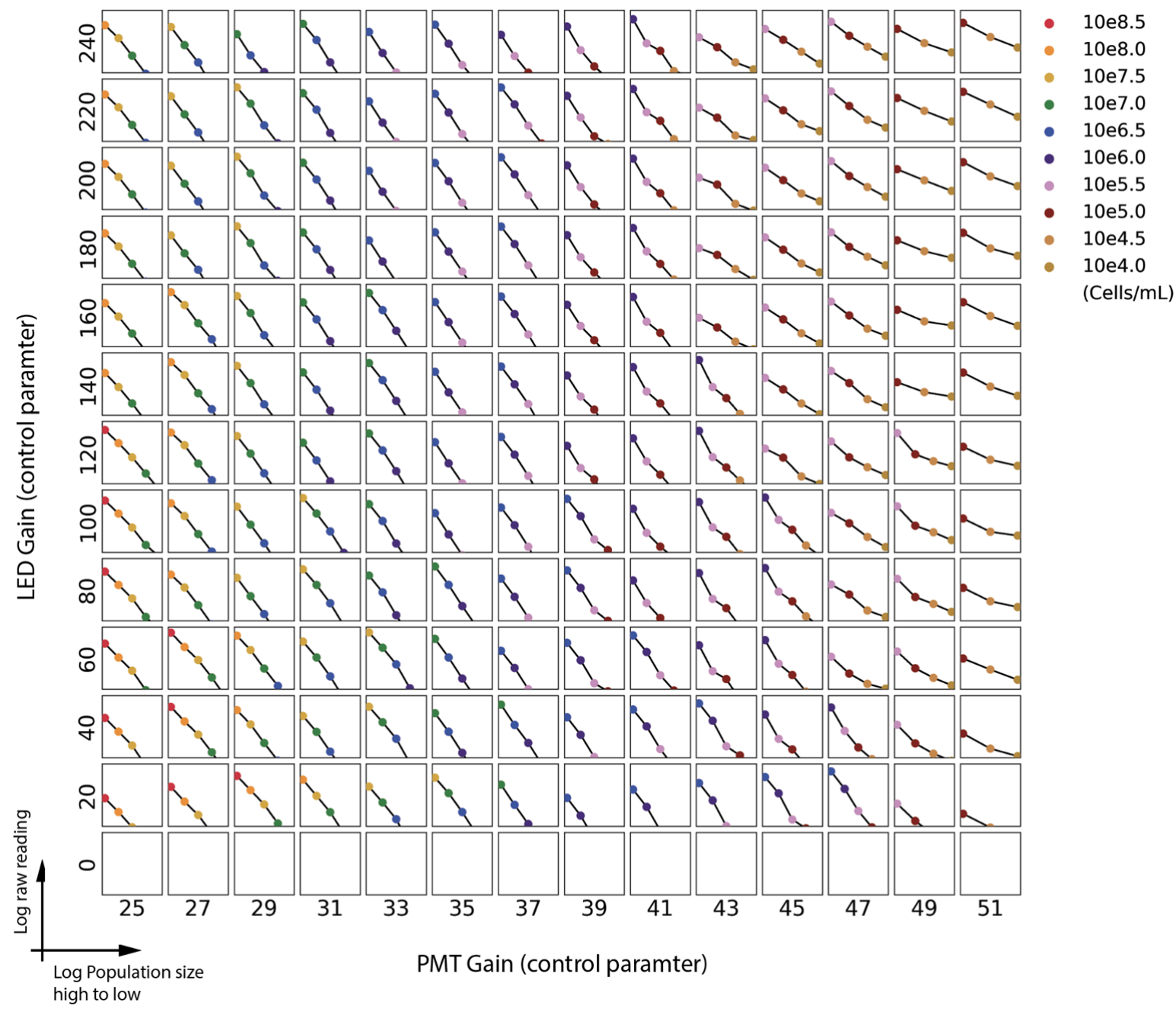

Figure S11. Map of log raw reading vs log sample's population size plots measured by different combinations of PMT and LED gains.

$\mathrm{x}$-axes in each subplot are log samples' population size while the $\mathrm{y}$-axes are the log readings. Colored dots indicate the reads gathered when measuring the samples with population densities indicated in the legend. 


\section{S-III. Selecting the proper filters for the multiple fluorophore monitoring module.}

When monitoring multiple fluorescent channels concurrently, the challenge is to limit crosscontamination of signal with a careful choice of fluorophores and filters. In this paper we showed an example of a proper set of filters/fluorophores selections for a two fluorophores monitoring setup with negligible levels of cross excitation/signal transmissions between channels. The characteristics of the chosen components are shown in Table S2. Theoretical spectral profiles of using these components in the fluorescence monitoring module are shown in Figure S12.

\begin{tabular}{|l|c|c|c|c|}
\hline & \multicolumn{2}{|c|}{ eGFP Channel } & \multicolumn{2}{c|}{ mKate Channel } \\
\hline & peak wavelength & bandwidth & peak wavelength & bandwidth \\
\hline Excitation light source & $470 \mathrm{~nm}$ & $25 \mathrm{~nm}$ & $590 \mathrm{~nm}$ & $15 \mathrm{~nm}$ \\
\hline Excitation filter & $469 \mathrm{~nm}$ & $35 \mathrm{~nm}$ & $586 \mathrm{~nm}$ & $20 \mathrm{~nm}$ \\
\hline Excitation dichroic beamsplitters & & & & \\
\hline Protein excitation spectrum & $470 \mathrm{~nm}$ & $588 \mathrm{~nm}$ & \\
\hline Protein emission spectrum & $507 \mathrm{~nm}$ & & $635 \mathrm{~mm}$ & \\
\hline Signal dichroic beamsplitters & & & & \\
\hline Signal filter & $525 \mathrm{~nm}$ & $15 \mathrm{~nm}$ & $640 \mathrm{~nm}$ & $14 \mathrm{~nm}$ \\
\hline
\end{tabular}

Table S2: List of filters/fluorophores selection for a two fluorophores monitoring setup. 


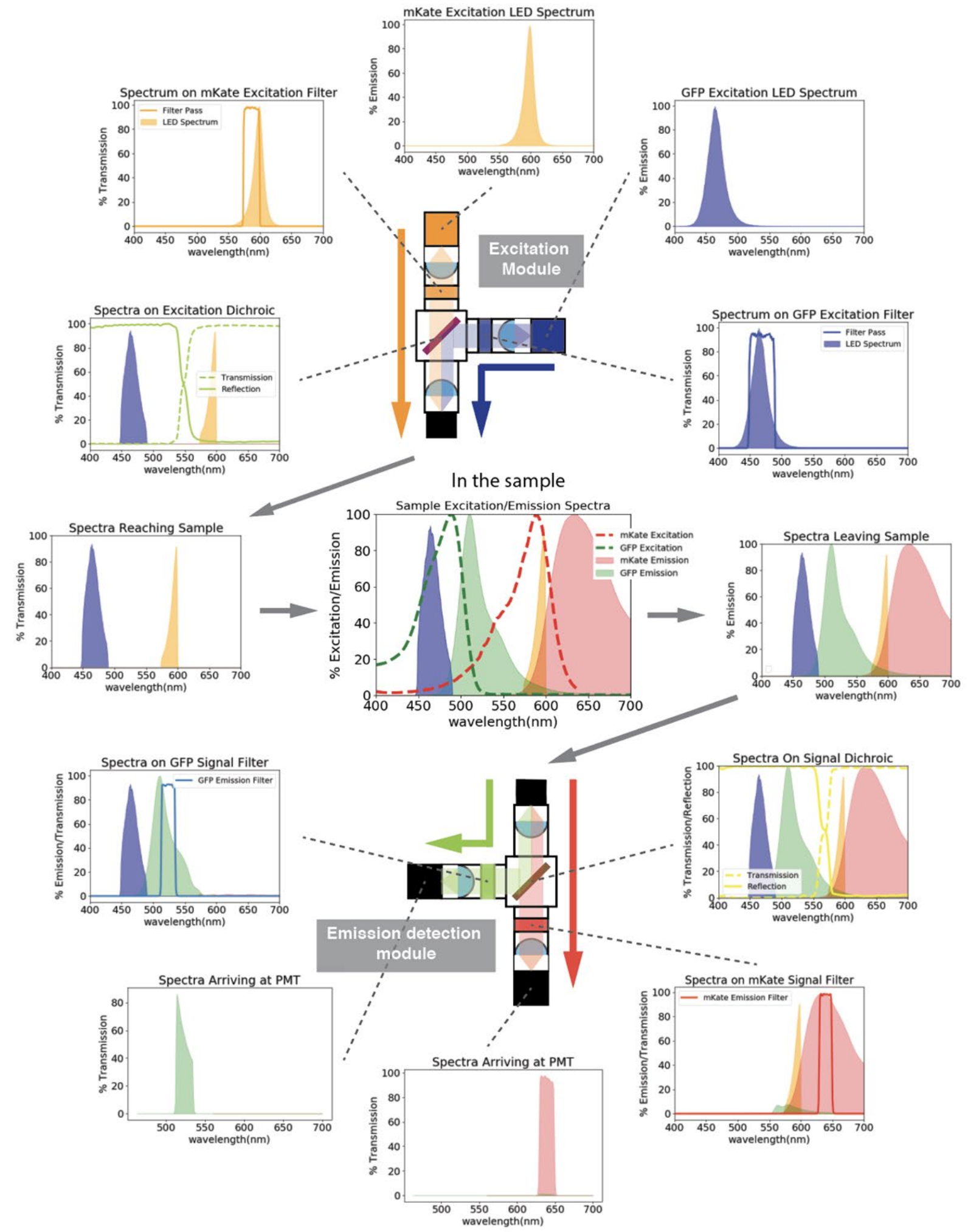

Figure S12. Theoretical spectral profiles of each component when applied in our two fluorophore monitoring setup. 


\section{S-IV. Feedback algorithm for expression level control}

The liquid pumps in our system are directed by a custom control algorithm which we can dynamically tune to specific needs of the experiment and sample conditions. For the example of the arabinose induced gene expression system, the feedback algorithm can be adjusted to automatically control two separated inlet pumps, with one of the inlet pumps connected to a bottle with pure M9 media while the other inlet pump is connected to a bottle containing M9 media saturated with arabinose. By changing the activation ratio of each pump, the arabinose concentration of the dilution media can be manipulated. Given the desired turbidity and the target per cell fluorescence expression level, the system is able to change the arabinose concentration based on the current per cell fluorescence expression level and $\mathrm{R}_{\mathrm{fdS}-\mathrm{fdOD}}$ to reach the target per cell fluorescence expression level. 


\section{S-V. Setup for experiments that require basic air handling.}

The culturing vials in our setup are locked in place and sealed by a gasket when running an experiment. This construction allows the vial lid to handle higher internal pressure. Thus, one can achieve anaerobic culturing or $\mathrm{CO}_{2}$ feeding in our setup by simply connecting a gas tank to our continuous culturing module. The gas tank creates a positive pressure that pushes the ambient air (and oxygen) out of the vials, and also prevents any air exchange from outside. The inlet media pump can operate at a higher frequency to compensate for an increased media evaporation rate due to the continuous gas input.

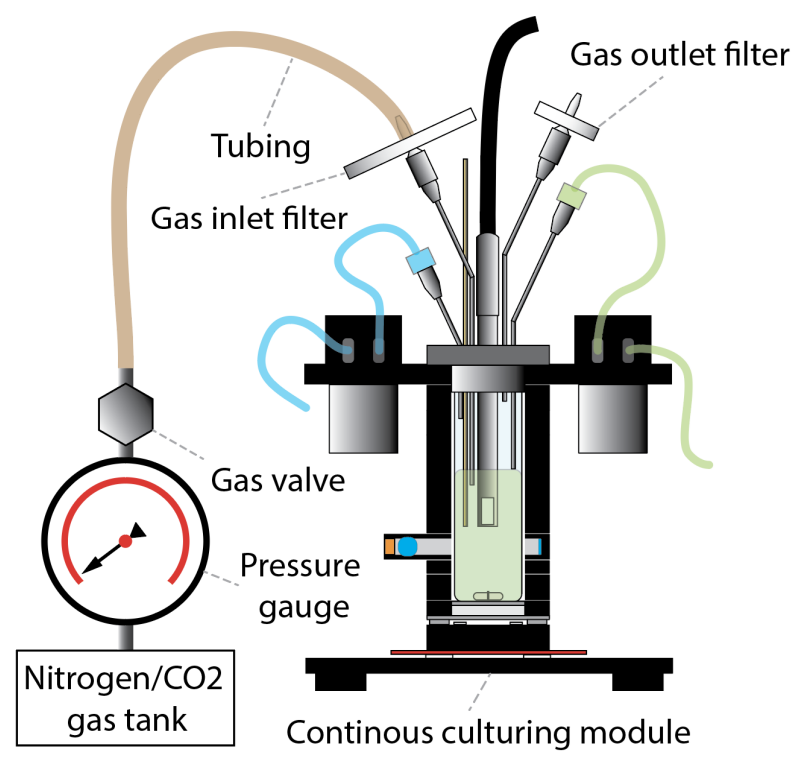

\section{S-VI. Device design documentation}

Device source code, PCB schematics, parts lists, and general documentation is publicly available at this github repository: https://github.com/ATCCMFM 